\section{Buchrezension zu: Einführung in die Organische Chemie}

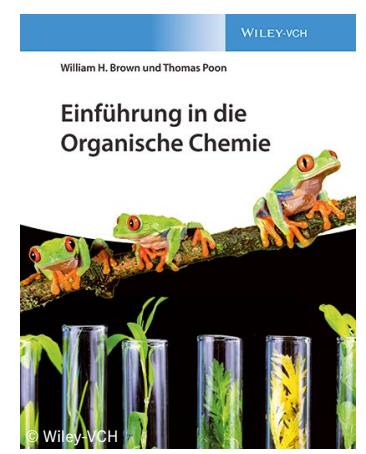

Einführung in die Organische Chemie

William H. Brown und Thomas Poon

734 S., 75 farb. Abb., Wiley-VCH, 2020. HC, 59,90€.

ISBN: 9783527346745

Auch als E-Book erhältlich

DOI: $10.1007 / \mathrm{s} 12268-021-1518-4$

(C) Springer-Verlag GmbH 2021

Ein Lehrbuch der Organischen Chemie hat mindestens furchterregende 1.500 Seiten. Eine Einführung in die Organische Chemie, wie die hier vorliegende mit ihren 734 Seiten, lebt daher von der ,Kunst des Weglassens', auch ,Mut zur Lücke' genannt.

Die Autoren Brown und Poon sind erfahrene Buchautoren und Hochschullehrer, die ein feines Gespür dafür haben, wie viel Chemie jeder Naturwissenschaftler eigentlich kennen und wissen sollte. Sie wenden sich ausdrücklich an Studenten der Biologie und der modernen Medizin, aber auch Ausbildungsgänge der Lebensmittelchemie, der Agro-, der Kunststoff- und der Verfahrenschemie sind ohne solide und breite Chemiekenntnisse nicht denkbar. Jeder Dozent der Gebiete, die man heute die Lebenswissenschaften nennt, wäre glücklich, wenn er auf diesen 734 Seiten Chemie aufbauen könnte.

Anschaulich, verständlich und grafisch übersichtlich wird der Leser interessiert und vereinnahmt. Vor allem zwei essenzielle Punkte werden hervorragend dargestellt - zum einen die Mecha- nismen der Reaktionen, zum anderen die Methoden, mit denen man sie aufklärt, vor allem die Infrarot- (IR-) und die Kernresonanz(NMR)-Spektroskopie.

Didaktisch hervorragend ist in Brown und Poons ,Einführung die Aufgabe gelöst, den Lernenden zu aktivieren: Immer wieder werden Aufgaben gestellt, leicht bis anspruchsvoll, spannend in jedem Fall. Einzigartig ist die Vielfalt der Didaktik: Jedes Kapitel wird mit einem Bild und Satz eröffnet, die zunächst unverständlich sind und neugierig machen. Jedes Kapitel endet außerdem mit einer knappen Zusammenfassung, in der die Fragestellung und die Schlüsselbegriffe durch Fettdruck hervorgehoben sind. Abgerundet wird die Zusammenfassung durch eine ,Quiz' genannte Serie von Aussagen, die man als richtig oder falsch klassifizieren soll. Doch auch damit noch nicht genug: Zu jedem Schlüsselbegriff gibt es ganz klassische Aufgaben, die leicht zu lösen sind, wenn man die vorausgehenden Abschnitte mit Fragestellungen, Vorgehensweisen und Antworten fleißig bearbeitet hat.

Und wenn es an Motivation fehlt, sich so umfangreich didaktisch bearbeiten zu lassen? Dann gibt es, blau unterlegt, Exkurse, interessante Ausflüge in Anwendungen des soeben Gelernten. So findet man z. B. in dem seiner $\mathrm{Na}$ tur nach etwas trockenen Kapitel der Halogenkohlenwasserstoffe einen Exkurs in die Chemie des Ozonlochs mit seinen lebensvernichtenden Folgen und der weltweiten Kooperation bei der Lösung der resultierenden Probleme.

Ohne Frage: ein modernes, sehr zu empfehlendes Lehrbuch!

Ferdinand Hucho,

Berlin,

hucho@chemie.fu-berlin.de 